\title{
Mobility Load Balancing Scheme Based on Cell Priority in Heterogeneous Cellular Networks
}

\author{
Lin Xiao ${ }^{1}$, Fahui $\mathrm{Wu}^{1}$, Xiaolan Zhang $^{2}$ and Tiankui Zhang ${ }^{2}$ \\ ${ }^{1}$ Information engineering School, Nanchang University \\ ${ }^{2}$ Beijing University of Posts and Telecommunications \\ xiaolin910@gmail.com; fahui97@126.com; \\ zxlbupt@126.com; tiankuizhang@qq.com
}

\begin{abstract}
Mobility load balancing (MLB) is a significant technology in Long Term Evolution Advanced heterogeneous cellular networks (HCN). To solve high handover performance loss issue and the potential Ping-Pong problem, a novel MLB scheme based on cell priority is proposed. Considering the impact of different user mobile speeds and cell coverage radius of $H C N$, we define a handover tolerance as the priority of load balancing to reduce the system performance loss caused by user handover. Furthermore, we use the load balancing index to evaluate the load balancing of HCN, then the load allocation mechanism of overlapping cells is deployed to overcome potential Ping-Pong problem. Simulation results show that the proposed MLB scheme has better performance than the original MLB scheme in terms of the system throughput and the number of unsatisfied users. Meanwhile, the handover times are also improved.
\end{abstract}

Keywords: heterogeneous cellular networks; mobility load balancing; handover tolerance; Ping-Pong; unsatisfied users

\section{Introduction}

In order to meet the fast growth of wireless communication data rates and higher coverage quality requirements, the Heterogeneous Cellular Networks (HCN) technology launched by Third Generation Partnership Project (3GPP) has been investigated intensely. HCN is able to improve system capacity as well as coverage efficiency by deploying additional small cellular base stations (BSs), i.e., pico BSs and femto BSs, under the coverage area of traditional macro cellular networks. However, the intensive deployment of small cellular BSs makes the mobility management unprecedentedly imperative.

In $\mathrm{HCN}$, the load imbalance result in the higher blocking probability in heavily loaded cells but the lower resource utilization in lightly loaded cells, which jeopardizes user satisfaction and undermines the system throughput. In order to solve these problems, mobility load balancing (MLB) technology is introduced. In the MLB implementation process, firstly, to achieve the current load information of other cells, the heavily loaded cell interacts with its neighboring lightly loaded cell through X2 interface. Then, the source cell with over-loaded traffic will select one potential target cell with under-loaded traffic to offload. The whole process is taken in an automatic way by setting the optimum handover offset value between the source cell and a possible target cell [1]. As defined in 3GPP TS 36.331, a handover can be triggered by a number of events [2]. In this paper, we are concerned with a particular event, known as event A3, which defines the entering and leaving conditions of handover. That is, the event is reached and reported only when the entering criterion is kept fulfilled and the leaving condition is not reached within the interval time-to-trigger. MLB is based on the entering condition. When the inequality of entering condition is hold for a particular UE, the UE will be handed over from its currently serving cell to a specific neighbor target cell [3]. 
Development, simulation and implementation of MLB have attracted a lot of research from both academic and industrial fields. In [4], a target cell selection algorithm in MLB is proposed, where both the traffic shifting request of the source cell the available spectrum of the neighboring lightly loaded cell are taken into consideration, when deciding the proper target cell. MLB in [5] can redistribute traffic load by changing cell-specific offset and consider its neighboring cells' load and the surrounding environments. A novel MLB scheme based two-step network flow is presented is presented in [6] which first optimizes the procedure of overload traffic transfer, and then considers capabilities of users when selecting the specific handover users. Authors in [7] proposed a Nash Bargaining Solution based user association scheme to improve the throughput of users and load balancing throughout the networks. In [8], a user relay assisted load balancing framework is proposed where the users from the high-loaded target cell can serve as relay for the shifted edge users from the heavy loaded source cell, thereby enhancing the throughput of the shifted users. However, all of the aforementioned approaches mainly focus on the methods of how to select the target cell with under-loaded traffic and improve the throughput of shifted users, ignoring the phenomenon that due to the insufficient load information of other cells, Ping-Pong problem is triggered [9] [10]. Ping-Pong problem means the offloading will be returned to the source cell again in a short period of time. What's more, related researches neglected the effect of the different users mobile speed and cell coverage radius of HCN to the system throughput and the load balancing.

In this paper, we propose a MLB scheme based on cell priority in HCN. The proposed scheme fully considers the impact of the different users mobile speeds and cell coverage radius of HCN. In order to reduce the system performance loss caused by user handover, we set handover tolerance as the priority of load balancing. Meanwhile, we propose the load allocation mechanism of overlapping cells to solve the potential Ping-Pong problem. Simulation results show that the scheme can improve system throughput performance, enhance user satisfaction and reduce the handover times.

The rest of this paper is organized as follow. Section 2 introduces the system model, including a $\mathrm{HCN}$ scenario and load balancing evaluation metrics; In Section 3, we describe the problems of load balancing. Section 4 gives the details of the proposed MLB; Simulation results are provided in Section 5; Section 6 concludes the work.

\section{System Model}

\subsection{A HCN Scenario}

Herein, we focus on the macro-pico scenario of HCN. Picocells deploy throughout the macrocell layout to provide better coverage and higher system capacity. As shown in Figure 1 , when a user moves, handover will happen. We use $N, K$ to denote the sets of BSs and users, respectively. For searching the user mobility, an assignment indicator variable $I_{i, k}(\mathrm{t})$ is defined corresponding to the association $\left(i . e ., I_{i, k}(\mathrm{t})=1\right.$ when user $k$ is associated with cell $i$ at time slot $t, I_{i, k}(\mathrm{t})=0$ otherwise) [11]. 


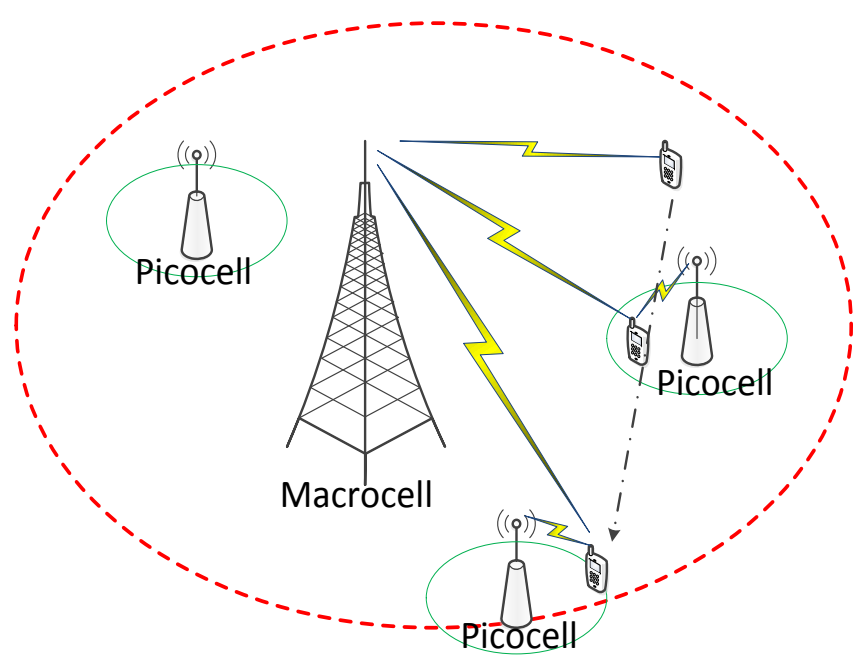

Figure 1. A HCN Scenario

When user $k$ is connected to the cell $i$, the received signal to interference plus noise ratio (SINR) is given by

$$
\operatorname{SINR}_{i, k}=\frac{P_{i} g_{i, k}}{\sum_{j \in N, j \neq i} \mathrm{P}_{j} g_{j, k}+\sigma^{2}}
$$

where $P_{i}$ is the transmit power of cell $i$ in one RB, $g_{i, k}$ indicates the link gain between cell $i$ and user $k$, which in general includes path loss, shadowing loss, penetrate loss and antenna gain, while fast fading is not considered here. $\sigma^{2}$ denotes the noise power. The corresponding bandwidth efficiency $r_{i, k}$ is

$$
r_{i, k}=B \log _{2}\left(1+\operatorname{SINR} R_{i, k}\right)
$$

The quantity of resources allocated to user $k$ by cell $i$ depends on its rate requirements $\theta_{k}$ and $r_{i, k}$, which is

$$
s_{i, k}=\frac{\theta_{k}}{r_{i, k}}
$$

\subsection{Load Balancing Evaluation Metrics}

We use $\rho_{i}$ to represent the load of cell $i$, which is:

$$
\rho_{i}=\frac{\sum_{k \in K} I_{i, k} s_{i, k}}{s}
$$

where $s$ denotes the total amount of PRBs for each cell. We define a load balancing factor to measure the degree of load balancing of the entire network as follows:

$$
\xi=\frac{\left(\sum_{i \in N} \rho_{i}\right)^{2}}{|N|\left[\sum_{i \in N}\left(\rho_{i}\right)^{2}\right]}
$$

where $|N|$ is the number of cells in the network, and the load balance index takes the value in the interval $[1 /|N|, 1]$. A larger $\xi$ means a more balance load distribution among cells. 


\section{Problem Formulation}

Figure 2 illustrates an implementation process of a conventional MLB. In MLB, each cell will adjust the handover regions by biasing the handover measurements. More specifically, for event A3, the handover condition can be expressed as [3]

$$
M_{j}-M_{i}>O_{i}^{(c s)}-O_{i, j}^{(c n)}+H y s+o f f
$$

where $M_{i}$ and $M_{j}$ correspond to the reference signal received power (RSRP) of cells $i$ and $j$, respectively. $O_{i}^{(c s)}$ is the cell-specific offset of the serving cell $i$, and $O_{i, j}^{(c n)}$ is the cellspecific offset of the neighbor cell $j$ with respect to cell $i$. Hys indicates a hysteresis term and off denotes a fixed offset. By increasing the offset $O_{i, j}^{(c n)}$, it is possible to cause a mobile user served by cell $i$ to be handed over to the neighbor cell $j$, thereby reducing the load in cell $i$.

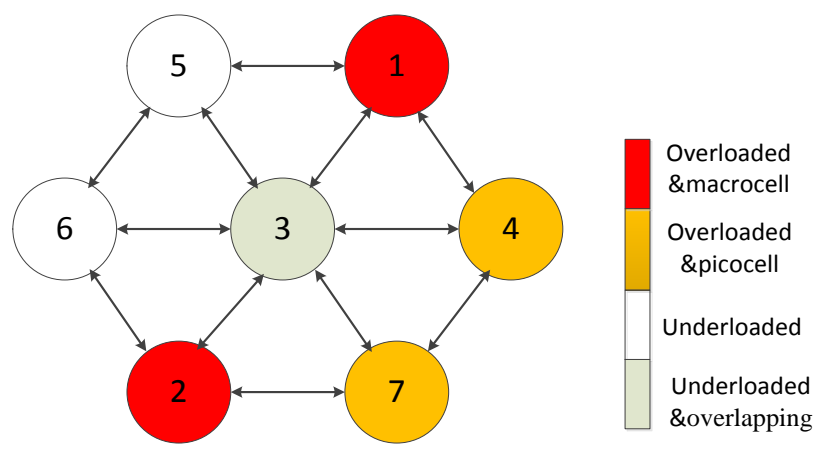

\section{Figure 2. One Implementation Use Case of a Conventional MLB}

In Figure 2, cell 1, cell 2 and cell 5 are macrocell and the other cells are picocell. At some time, cell 1 is over-loaded. If using the conventional MLB, cell 1 will interact its current load information with its around adjacent cells. Meanwhile, cell 3 and cell 5 can be selected as the offloading target cells. However, at the same time, cell 2 is also over-loaded, in a similar way, cell 3 and cell 6 can be selected as the offloading target cells. Due to the insufficient load information of other cells, cell 1 and cell 2 transferring load towards cell 3 may result in the over load of cell 3 and also the Ping-Pong problem between cell 3 and cell 1 . In addition, there is another problem of cell 1 to select the offloading target cell due to the type difference between cell 3 and cell 5. To solve both the Ping-Pong and cell selection problem, we propose a MLB scheme based on cell priority.

\section{Proposed MLB Scheme}

In this part, we set handover tolerance as the priority of cell selection to solve the problem of handover performance loss. Then, in the process of load balancing, if there are the phenomenon of potential overlapping cells and Ping-Pong problem, a load allocation mechanism is proposed. In addition, the final threshold of load balancing is defined to judge the load balancing level. At last, the procedure of the proposed MLB is presented.

\subsection{Handover Tolerance}

In the process of MLB, the different user mobile speeds and cell coverage radius of HCN lead to a system performance loss, which seriously affect the load balance. In this paper, a handover tolerance is defined to reduce the system performance loss caused by user handover. In $\mathrm{HCN}$, macrocell coverage is greater than the picocell, so there is no significant difference performance in two areas when the UE moves slowly. However, due to the limit of picocell 
coverage, it is more easily to incur Ping-Pong effect with high speed, so the macrocell has higher handover tolerance, in other words, the system performance loss is less in macrocell. In addition, when the cell load changes less drastically, the handover tolerance would become bigger. Based on the previous analysis, we can conclude:

$$
\begin{aligned}
T_{i} & \propto R_{i} \\
T_{i} & \propto \frac{1}{v_{i}} \\
T_{i} & \propto \frac{1}{l_{i}}
\end{aligned}
$$

where $T_{i}$ is the handover tolerance of cell $i, R_{i}$ and $l_{i}$ denote the cell coverage radius and load value of cell $i$, respectively. $v_{i}$ indicates user mobile speed in cell $i$. $T_{i}$ can be simplified in the appropriate range as:

$$
T_{i}=\frac{\frac{R_{i}}{\sum R_{i}}}{l_{i}+\frac{v_{i}}{v_{\max }}}
$$

According to (8), heavily loaded cell can calculate the handover tolerance and priority according to the load value, and then select the lightly loaded cell in turn to achieve the purpose of reducing the system performance loss.

\subsection{Load Allocation Mechanism of Overlapping Cells}

In order to solve the Ping-Pong problem, we assume that the special network area $c$ have $M$ heavily loaded cells, at the same time the overlapping lightly loaded cells are $P$, then the load allocation mechanism of overlapping cells can be shown below. According to load balancing factor (5), our goal is to maximize load balancing index in the special area.

$$
\max \xi_{M}=\max \sum_{i=1}^{M} \xi_{i}=\max \sum_{i=1}^{M} \frac{\left(L_{i}+a_{i}\right)^{2}}{|N| \sum_{c}\left(\rho_{i}\right)^{2}}
$$

where $\xi_{i}$ is the load balancing factor composed of a heavily loaded cell $i$ and its corresponding lightly loaded cells, $L_{i}$ is the load of heavily loaded cell $i$ and its corresponding lightly loaded cells, $a_{i}$ denotes the load assigned to cell $i$, which can be a lightly loaded cell or multiple lightly loaded cells.

As the denominator of (9) is a constant value, so the optimization problem can be converted as:

$$
\begin{aligned}
\max \sum_{i=1}^{M}\left(L_{i}+a_{j}\right)^{2} & =\sum_{i=1}^{M}\left(L_{i}^{2}+2 a_{i} L_{i}+a_{i}^{2}\right) \\
& =\sum_{i=1}^{M} L_{i}^{2}+\sum_{i=1}^{M}\left(2 a_{i} L_{i}+a_{i}^{2}\right) \\
& =\sum_{i=1}^{M} L_{i}^{2}+2\left(a_{1} L_{1}+L+a_{M} L_{M}\right)+\left(a_{1}^{2}+L+a_{M}^{2}\right)
\end{aligned}
$$

By sorting $L_{i}$, we assume that $L_{1} \geq L_{2} \geq L_{3} \cdots \geq L_{M}$, the load value of overlapping lightly loaded cells is $\tilde{A}$. We further have: 


$$
\begin{aligned}
& \max \sum_{i=1}^{M}\left(\mathrm{~L}_{i}+\mathrm{a}_{j}\right)^{2} \\
& =\sum_{i=1}^{M} L_{i}^{2}+2\left[L_{1}\left(\tilde{A}-a_{2}-a_{3}-\cdots-a_{M}\right)+L_{2} a_{2}+\cdots+L_{M} a_{M}\right]+\cdots+\left(\mathrm{a}_{1}^{2}+\mathrm{a}_{2}^{2}+\cdots+\mathrm{a}_{M}^{2}\right) \\
& \approx \sum_{i=1}^{M} L_{i}^{2}+2 L_{1} \tilde{A}+\left(\mathrm{a}_{1}^{2}+\cdots+\mathrm{a}_{M}^{2}\right)-2 \sum_{i=2}^{M} a_{i} \\
& =\tilde{L}-2 a_{1}\left(a_{2}+\cdots+a_{M}\right)-2 a_{2}\left(a_{3}+\cdots+a_{M}\right)-\cdots-2 a_{M-1} a_{M}-2 \sum_{i=2}^{M} a_{i}
\end{aligned}
$$

In (11), $\tilde{L}=\sum_{i=1}^{M} L_{i}^{2}+2 L_{1} \tilde{A}+\tilde{A}^{2}$, which is a constant value. Then, $\sum_{i=1}^{M} a_{i}$ is divided into two categories, $a_{1}$ and $\sum_{i=2}^{M} a_{i}$, so the load allocation mechanism of overlapping cells can be divided into following three cases:

Case 1: when $a_{1}$ is 0 and $\sum_{i=2}^{M} a_{i}$ is $\Delta L$, the load balancing index is $\tilde{L}-2 a_{2}\left(a_{3}+\cdots+a_{M}\right)-\cdots-2 a_{M-1} a_{M}-2 \sum_{i=2}^{M} a_{i}$;

Case 2: when $a_{1}$ is $\Delta L$ and $\sum_{i=2}^{M} a_{i}$ is 0 , the load balancing index is $\tilde{L}$;

Case 3: when $a_{1}$ is the value of any one or more lightly loaded cells and $\sum_{i=2}^{M} a_{i}$ is $\Delta L-a_{1}$, the load balancing index is $\tilde{L}-2 a_{1}\left(a_{2}+a_{3}+\cdots+a_{M}\right)-2 a_{2}\left(a_{3}+\cdots+a_{M}\right)-\cdots-2 a_{M-1} a_{M}-2 \sum_{i=2}^{M} a_{i}$.

We can conclude that when the load allocation mechanism of overlapping cells satisfies the case 2, we can maximize the load balancing index in the special network. When the network have heavily loaded cells and overlapping lightly loaded cells, the overlapping lightly loaded cells are all assigned to the heaviest load area, which can achieve the optimal load allocation.

\subsection{Final Threshold of Load Balancing}

In this part, we put forward a method, which adopts the final threshold to quantify the load transfer and determine the load balancing level. According to function (5), the value of objective function after handover should be larger than that before handover. Assuming user $k$ is switched from its original cell $i$ to target cell $j$, we can derive:

$$
\begin{gathered}
\xi_{i, k}=\frac{\left(\rho_{i}+\rho_{j}\right)^{2}}{2\left(\rho_{i}^{2}+\rho_{j}^{2}\right)} \\
\xi_{j, k}=\frac{\left[\left(\rho_{i}-\Delta s_{i, k}\right)+\left(\rho_{j}+\Delta s_{j, k}\right)\right]^{2}}{2\left[\left(\rho_{i}-\Delta s_{i, k}\right)^{2}+\left(\rho_{j}+\Delta s_{j, k}\right)^{2}\right]} .
\end{gathered}
$$

In general, the user $k$ has approximately the same load value in cell $i$ and cell $j$, i.e., $\Delta s_{i, \mathrm{k}} \approx \Delta s_{\mathrm{j}, \mathrm{k}}$, and then the function $\xi_{j, k}>\xi_{\mathrm{i}, k}$ can be converted as

$$
\max \left\{\left(\rho_{i}^{2}+\rho_{j}^{2}\right)-\left[\left(\rho_{i}-\Delta s_{i, k}\right)^{2}+\left(\rho_{j}+\Delta s_{j, k}\right)^{2}\right]\right\}
$$

In order to calculate conveniently, we replace $\rho_{i}{ }^{\prime}$ with $\rho_{i}-\Delta s_{i, k}$ and replace $\rho_{j}{ }^{\prime}$ with $\rho_{j}+\Delta s_{\mathrm{j}, k}$, and we obtain 


$$
\begin{aligned}
& \max \left[\left(\rho_{i}^{2}+\rho_{j}^{2}\right)-\left(\rho_{i}{ }^{\prime 2}+\rho_{j}{ }^{\prime 2}\right)\right] \\
& \text { s.t. }\left\{\begin{array}{l}
\rho_{i}{ }^{\prime}+\rho_{j}{ }^{\prime}=\rho_{i}+\rho_{j} \\
\rho_{j}{ }^{\prime} \leq \rho_{i}{ }^{\prime} \leq \rho_{i} \\
\rho_{j} \leq \rho_{j}{ }^{\prime} \leq \rho_{\text {threshold }}
\end{array}\right.
\end{aligned}
$$

where $\rho_{\text {threshold }}$ represents the final threshold of load balancing. Combined with (15), when $\rho_{i}{ }^{\prime}=\rho_{j}{ }^{\prime}=\frac{\rho_{i}+\rho_{j}}{2}$, the maximum value can be attained. After load balancing, heavily loaded cell and lightly loaded cell reach approximately the same load value (defined as the final threshold), and the load is average of the total value before load balancing.

\subsection{Description of the Proposed MLB}

The steps of the proposed MLB scheme are summarized as follows:

(1) In one specific network region, each cell $i$ should do the following feasibility judgments: if $\rho_{i}^{*}>\rho_{t h}$, the cell $i$ is judged as heavily loaded cell and the load balancing is triggered, where $\rho_{t h}$ is the trigger threshold of load balancing. Then, heavily loaded cells detect their adjacent cells and generate the corresponding load transfer list.

(2) Each cell $i$ calculates handover tolerance through (8) and sorts it to decide the transfer cell. Each cell $i$ would select a transfer cell with maximum handover tolerance. For the cell $i$, if such transfer cell exists, this scheme will further implement load balancing and turn to (3); otherwise, the cell $i$ try select a transfer cell with the second maximum handover tolerance.

(3) When overlapping lightly loaded cells exists in the network, it means the load allocation mechanism is triggered. After that, update the load transfer list and turn to (4).

(4) If the load of heavily load cells are all less than $\rho_{\text {threshold }}$, then the proposed MLB implements successfully. Otherwise, go to step (5).

(5) Each cell $i$ deletes the current transfer cell from the load transfer list and determines whether the load transfer list is empty. If it is, then wait for the next period. Otherwise, back to (2) and choose the next transfer cell.

\section{Simulation Result}

\subsection{Simulation Parameters}

Under the simulation scenario which is formed by 7 hexagonal cells with two tiers of wrap-around setup, we evaluate the performance of the proposed MLB scheme in HCN. For the sake of simplicity and without loss of generality, effects of RLC, HARQ and physical layer are omitted here. Detailed system parameters are given in Table 1.

Table 1. Parameters of Simulation

\begin{tabular}{|c|c|}
\hline Parameter & Value \\
\hline Macro cell layout & $7 \times 3$ sectors, hexagonal \\
\hline Pico cells per macro & 4 \\
\hline Macro cell Radius & $500 / \sqrt{3}$ meters \\
\hline Carrier frequency & $2.0 \mathrm{GHz}$ \\
\hline Simulation Time & $100 \mathrm{~min}$ \\
\hline System bandwidth & $10 \mathrm{MHz}$ \\
\hline
\end{tabular}




\begin{tabular}{|c|c|}
\hline Transmission power & $\begin{array}{c}46 \mathrm{dBm} \text { Macro cells } \\
30 \mathrm{dBm} \text { Pico cells }\end{array}$ \\
\hline Fading channel model & Typical Urban \\
\hline Total UE number in each sector & 60 \\
\hline Clustered UE number in each picocell & 10 \\
\hline
\end{tabular}

\subsection{Performance Comparison and Analysis}

Conventional performance indexes, including handover times, system throughput and the number of unsatisfied users, are selected to validate the efficiency and effectiveness of the proposed MLB scheme. There are two reference scenarios, conventional load balancing (CLB) presented in [11] and a LB method based on cell association (CA-MLB) presented in [12].

Figure 3 shows the handover times versus load balancing times (simulations times). The value is supposed to be the lower the better, for the reason that handover times affect service quality and user experience. We can observe that there are less handover times using the proposed MLB than the other two methods, for the reason that it adopts handover tolerance and load allocation mechanism of overlapping cells to reduce the Ping-Pong problem. Accordingly, the system performance loss has reduced.

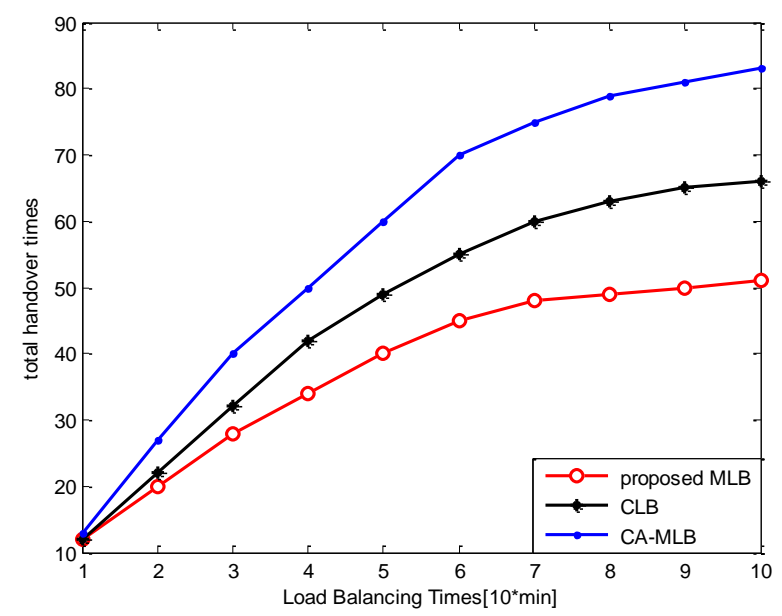

Figure 3. Handover Times versus Load Balancing Times

In overloaded cells, part of the users cannot fulfill their service requirement, which is defined as unsatisfied users. For cell ${ }^{c}$, the total required number of PRBs and the amount of the total available PRBs are $N_{r}$ and $N_{a}$ respectively. The number of users in cell $c$ is represented as $Q_{c}$. Thus, the number of unsatisfied users is written as $z=\sum_{\forall c} \max \left(0, Q_{c} *\left(1-\frac{N_{a}}{N_{r}}\right)\right)$. The detailed definition of unsatisfied users is shown in [5].

Figure 4 demonstrates the number of unsatisfied users versus load balancing times. It is obvious that the proposed MLB has a substantial improvement of the number of unsatisfied users over the other two methods.

Figure 5 is the statistical results of system throughput with load balancing times. It is shown that the proposed MLB can achieve a better system throughput compared with the other two methods. And after load balancing, due to the improvement of user satisfaction, some of the previous unsatisfied user's data can be transmitted normally, so that more users can access the proposed MLB scheme, which further achieves higher throughput. 


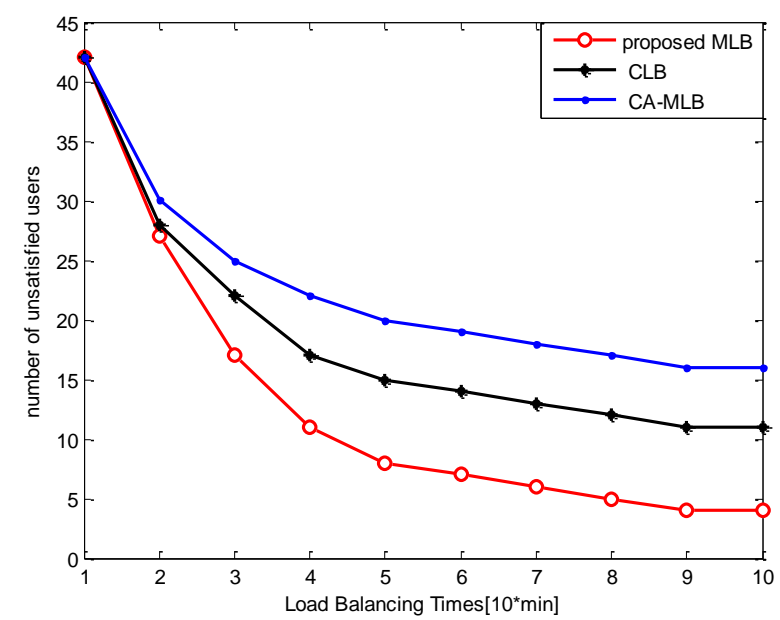

Figure 4. Number of Unsatisfied Users versus Load Balancing Times

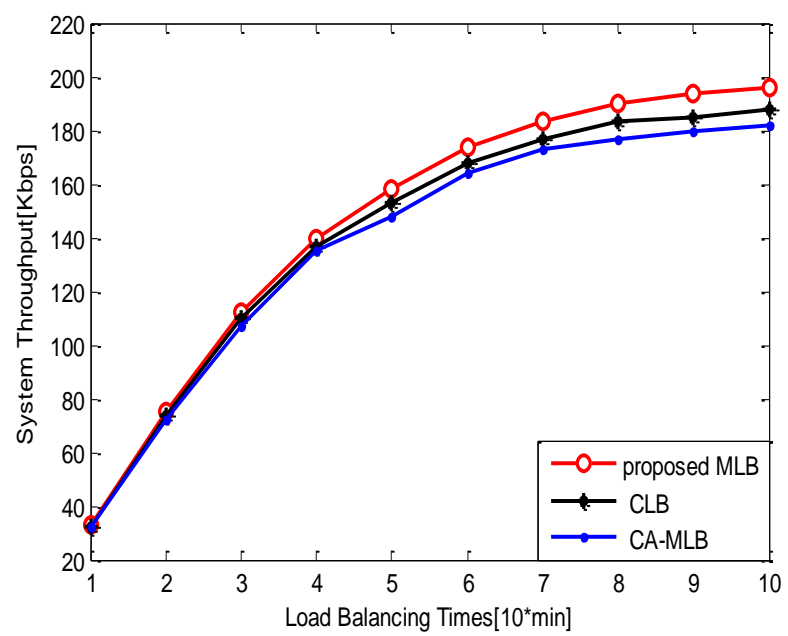

Figure 5. System Throughput Versus Load Balancing Times

\section{Conclusions}

To reduce system performance loss and solve the Ping-Pong problem, we proposed a MLB scheme based on cell priority, which is composed of cell selection algorithm and load allocation mechanism of overlapping cells. The handover tolerance is defined in the proposed MLB to select the transfer cell for the purpose of reducing system performance loss caused by user handover. The irrational load transferring problem can be solved efficiently by taking the hidden cells into consideration and implementing the optimal load redistribution. Therefore, Ping-Pong effects can be efficiently mitigated. Simulation results show that, on the premise of guaranteeing the system throughput, the proposed MLB scheme dramatically reduces the handover times and the amount of the unsatisfied users of the system. 


\section{References}

[1] S. Corroy, L. Falconetti and R. Mathar, "Dynamic cell association for downlink sum rate maximization in multi-cell heterogeneous networks", IEEE International Conference on Communications (ICC), (2012), pp. 2457-2461.

[2] 3GPP TS36.331 V10.1.0, Radio resource control (RRC), (2011).

[3] M. Sheng, C. G. Yang, Y. Zhang and J. D. Li, "Zone-based load balancing in LTE self-optimizing networks: a game theoretic approach", IEEE Transactions on Vehicular Technology, vol. 63, no. 6, (2014), pp. 29162925.

[4] L. Xu, Y. Chen, K. K. Chai, Y. Luan and D. Liu, "Cooperative mobility load balancing in relay cellular networks", Communications in China (ICCC), 2013 IEEE/CIC International Conference on, vol., no., (2013), pp.141, 146, 12-14.

[5] Y. Yang, P. F. Li and W. D. Wang, "Algorithm about mobility load balance considering system overhead on LTE system", IEEE Wireless and Optical Communication Conference (WOCC), (2013), pp. 231-235.

[6] D. Liu, Y. Chen, K. K. Chai, T. Zhang and M. Elkashlan, "Opportunistic User Association for Multi-service HetNets Using Nash Bargaining Solution”, IEEE Communications Letters, vol.18, no.3, (2014), pp.463, 466.

[7] D. Liu, Y. Chen, T. Zhang, K. K. Chai, J. Loo and A. Vinel, "Stackelberg Game Based Cooperative User Relay Assisted Load Balancing in Cellular Networks", IEEE Communications Letters, vol.17, no.2, (2013), pp.424,427.

[8] M. Dottling and I. Viering," Challenges in mobile network operation: towards self-optimizing networks", IEEE Int'l. Conf. Acoustics, Speech, and Sig. Processing, (2009).

[9] R3-101477, CMCC, Requirement for further enhancement of MLB, 3GPP TSG-RAN WG3 Meeting \#68 meeting, 10-14th May,Montreal, Canada, (2010).

[10] R3-102107, CMCC, An enhancement for MLB, 3GPP TSG-RAN WG3. Meeting \#69 meeting, 23 - 27th August, Madrid, Spain, (2010).

[11] Y. Bejeran and H. Seung-jae, "Cell breathing techniques for load balancing in wireless LANs", IEEE Transactions on Mobile Computing, vol. 8, no. 6, (2009), pp. 735-749.

[12] W.H. Lv, W.J. Li, H. Zhang and Y.G. Liu, "Distribution mobility load balancing with RRM in LTE", IEEE International Conference on Broadband Network and Multimedia Technology (ICBNMT), (2010). 